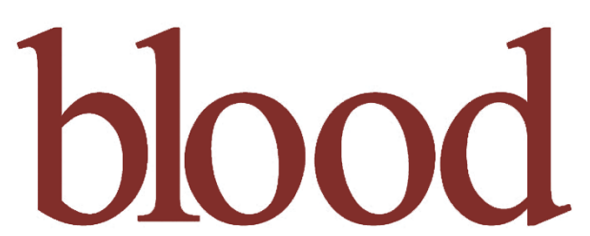

Prepublished online October 1, 2012;

doi:10.1182/blood-2012-04-425959

\title{
S1P1 expression is controlled by the pro-oxidant activity of p66Shc and is impaired in B-CLL patients with unfavorable prognosis
}

Nagaja Capitani, Laura Patrussi, Livio Trentin, Orso Maria Lucherini, Enrica Cannizzaro, Enrica Migliaccio, Federica Frezzato, Cristina Gattazzo, Francesco Forconi, Piergiuseppe Pelicci, Gianpietro Semenzato and Cosima T. Baldari

Information about reproducing this article in parts or in its entirety may be found online at: http://bloodjournal.hematologylibrary.org/site/misc/rights.xhtml\#repub_requests

Information about ordering reprints may be found online at:

http://bloodjournal.hematologylibrary.org/site/misc/rights.xhtml\#reprints

Information about subscriptions and ASH membership may be found online at: http://bloodjournal.hematologylibrary.org/site/subscriptions/index.xhtml

Advance online articles have been peer reviewed and accepted for publication but have not yet appeared in the paper journal (edited, typeset versions may be posted when available prior to final publication). Advance online articles are citable and establish publication priority; they are indexed by PubMed from initial publication. Citations to Advance online articles must include the digital object identifier (DOls) and date of initial publication.

Blood (print ISSN 0006-4971, online ISSN 1528-0020), is published weekly by the American Society of Hematology, 2021 L St, NW, Suite 900, Washington DC 20036.

Copyright 2011 by The American Society of Hematology; all rights reserved.

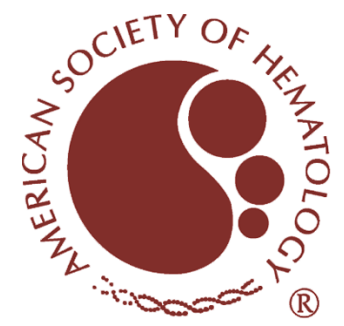




\section{S1P1 expression is controlled by the pro-oxidant activity of p66Shc and is impaired in B-}

\section{CLL patients with unfavourable prognosis}

Nagaja Capitani ${ }^{1,2^{*}}$, Laura Patrussi ${ }^{1,2^{*}}$, Livio Trentin ${ }^{3,4}$, Orso Maria Lucherini ${ }^{1,2}$, Enrica

Cannizzaro $^{1,2}$, Enrica Migliaccio ${ }^{5}$, Federica Frezzato ${ }^{3,4}$, Cristina Gattazzo ${ }^{3,4}$, Francesco

Forconi $^{6,7}$, Piergiuseppe Pelicci ${ }^{5}$, Gianpietro Semenzato ${ }^{3,4}$, Cosima T. Baldari $^{1,2}$

${ }^{1}$ Department of Evolutionary Biology and ${ }^{2}$ Istituto Toscano Tumori, University of Siena, Italy, University of Siena, Italy; ${ }^{3}$ Venetian Institute of Molecular Medicine, Centro di Eccellenza per la Ricerca Biomedica, Padua, Italy; ${ }^{4}$ Department of Medicine, Hematology and Clinical Immunology Branch, Padua University School of Medicine, Padua, Italy; ${ }^{5}$ Department of Molecular Oncology, European Institute of Oncology, Via Ripamonti 435, 20139 Milan, Italy;

${ }^{6}$ Department of Human Pathology and Oncology, University of Siena, Italy; ${ }^{7}$ Southampton General Hospital, Southampton, UK.

Corresponding author: Dr. Cosima T. Baldari, Department of Evolutionary Biology, Via Aldo Moro 2, 53100 Siena, Italy. Tel +39-0577-234400; Fax +39-0577-234476; email baldari@unisi.it

*Equal contribution

Running head: S1P receptor deficiency in CLL 


\section{ABSTRACT}

Although intrinsic apoptosis defects are causal to the extended survival of chronic lymphocytic leukemia (CLL) B cells, several lines of evidence support a contribution of the peripheral lymphoid organs and bone marrow microenvironment to the extended lifespan of leukemic B cells. Lymphocyte trafficking is controlled by homing signals, provided by stromal cell-derived chemokines, and egress signals provided by sphingosine-1-phosphate (S1P). We show that expression of S1P1, the S1P receptor responsible for lymphocyte egress, is selectively reduced in CLL B cells with unmutated IGHV. Expression of S1P2, which controls B-cell homeostasis, is also impaired in CLL B cells but independently of the IGHV mutational status. We provide evidence that p66Shc, a Shc adaptor family member whose deficiency is implicated in the apoptosis defects of CLL B cells, controls S1P1 expression through its pro-oxidant activity. p66Shc also controls the expression of the homing receptor CCR7, which opposes S1P1 by promoting lymphocyte retention in peripheral lymphoid organs. The data provide insights into the regulation of S1P1 expression in B cells and suggest that defective egress caused by the impaired S1P1 expression contributes to the extended survival of CLL B cells by prolonging their residency in the pro-survival niche of peripheral lymphoid organs. 


\section{INTRODUCTION}

Although progressive accumulation of monoclonal $\mathrm{CD} 5^{+} \mathrm{B}$ cells in blood, peripheral lymphoid organs and bone marrow is the hallmark of chronic lymphocytic leukemia (CLL), the clinical course of this disorder is highly variable, ranging from a stable disease that may only require monitoring over the time to a progressive, severe disease. ${ }^{1,2}$ A number of markers have been associated with poor prognosis. Coupled to the cytogenetic abnormalities, the mutational status of immunoglobulin heavy chain variable region (IGHV) genes is the most valuable marker presently available, with unmutated IGHV found in patients who develop aggressive disease. ${ }^{3}$ Nevertheless the onset of disease progression, as well as the response to treatment, are to date largely unpredictable.

At variance with other hematological malignancies, CLL B cells are usually arrested at $\mathrm{G}_{0} / \mathrm{G}_{1}$ and their accumulation is the result of an abnormally prolonged survival rather than uncontrolled proliferation. ${ }^{1,2}$ Intrinsic defects in the apoptotic machinery underlie the prolonged lifespan of CLL B cells, a major target being the Bcl-2 family, where overexpression of anti-apoptotic members (Bcl-2, $\mathrm{Mcl}-1)$ or impaired expression of proapoptotic members (Bax, Bak) tilts the balance towards cell survival. ${ }^{4}$ Extrinsic factors, consisting mainly of stromal cell-derived chemokines (CXCL12, CXCL13, CCL19, CCL21), also to contribute to the extended lifespan of CLL B cells by providing survival cues during their transit through peripheral lymphoid tissues and bone marrow. ${ }^{5}$ 
Lymphocyte trafficking is tightly controlled by the chemokines present in the lymphoid microenvironment and the chemokine receptors expressed by the lymphocyte itself. ${ }^{6}$ CLL B cells express increased levels of CXCR4, CCR7 and CXCR5, which has been proposed to enhance their homing to bone marrow and peripheral lymphoid tissues, thereby favouring cognate interactions with resident cells. ${ }^{7}$ In this context, unique features of CLL B cells with unmutated IGHV are an increased ability to phosphorylate p72Syk in response to slgM ligation ${ }^{8}$ and robust signaling by B-cell antigen receptors, ${ }^{9}$ which have been demonstrated to broadly recognize self-antigens such as modified cytoskeletal proteins and oxidation-specific epitopes. ${ }^{10,11}$ Hence the enhanced homing to the stromal microenvironment contributes to disease progression by favouring not only survival signaling but also mitogenic signaling by the B-cell antigen receptor in response to self-antigen presented by stromal cells, ${ }^{10}$ which is likely to account for the small proliferative fraction in CLL clones. ${ }^{3}$

Sphingosine-1-phosphate (S1P), a sphingolipid metabolite produced by sphingosine kinase- 1 and -2 and acting through the activation of the G-protein coupled receptors S1P1-S1P5, has recently emerged as a major player in lymphocyte trafficking. ${ }^{12}$ Extracellular S1P, largely derived from erythrocytes and lymphatic endothelial cells, accumulates at high concentrations (high $\mathrm{nM}-\mu \mathrm{M})$ in circulatory fluids. ${ }^{13}$ At variance, the interstitial S1P levels are maintained very low by a battery of S1P-degrading enzymes. ${ }^{14}$ This results in a gradient that promotes the egress of lymphocytes from their homing sites and their re-entry into the bloodstream or lymph, a process mainly controlled by 
S1P1. ${ }^{15}$ Hence the dwell time of lymphocytes in peripheral lymphoid tissues is tightly controlled by the opposing activities of chemokine and S1P receptors.

We hypothesized that, in addition to their enhanced homing due to increased expression of chemokine receptors, ${ }^{7}$ a defective response of CLL B cells to S1P would lead to an extended transit time in lymphoid tissues, thereby further favouring their interaction with the pro-survival stromal microenvironment. Here we show that neoplastic B cells from CLL patients with unmutated IGHV, but not from patients with mutated IGHV, have a profound deficiency in S1P1. We also show that the S1P1 defect, which is associated with an upregulation of CCR7, is selectively caused by an impairment in the expression of the pro-oxidant Shc family adaptor, p66Shc. 


\section{MATERIALS AND METHODS}

\section{Patients and healthy donors}

Blood samples were collected from 78 patients who satisfied standard morphologic and immunophenotypic criteria for CLL. Normal B cells from 13 buffy coats were used as control of the adult healthy population. Informed consent was obtained from all patients according to the Declaration of Helsinki. All specimens were collected from patients attending the Hematology and Clinical Immunology Branch, Padua University School of Medicine, from 2003 to 2012. At the time of the collection, patients had never received treatment. The research was approved by the local Ethics Committee.

B cells $\left(C D 19^{+}\right)$were purified from by negative selection using the RosetteSep Human B-cell enrichment Cocktail (StemCell Technologies Inc, Vancouver, Canada) followed by density gradient centrifugation on Lympholite (Cedarlane Laboratories Ltd, The Netherlands). The purity of the resulting B-cell population was $>90 \%$, as assessed by flow cytometry.

\section{Mice}

p66Shc $^{-/}$mice in the 129 genetic background were previously described. ${ }^{16}$ The work was carried out on the p66Shc ${ }^{-/-}$and 129 mouse colonies bred in the animal facility at the University of Siena. Analyses were performed on age- and sex-matched 2-9 monthold mice. All animal experiments were carried out in agreement with the Guiding Principles for Research Involving Animals and Human Beings and approved by the local ethics committee. 
Mice were sacrificed by cervical dislocation and spleen, lymph nodes, blood and bone marrow were harvested. In some experiments, splenic B or T cells were negatively purified by immunomagnetic sorting using either the Dynabeads Mouse CD43 or the Dynal Mouse T-cell Negative Isolation Kit (Invitrogen srl, Leek, The Netherlands), respectively, which resulted in $>85 \%$ purity, as assessed by flow cytometry.

\section{Cell lines, transfections and immunoblots}

The CLL-derived B-cell line MEC ${ }^{17}$ was used for the generation of stable transfectants. A mammalian expression vector (pcDNA3, Invitrogen srl, Leek, Netherlands) encoding human full-length p66Shc and the respective mutants p66ShcSA $(S \rightarrow A$ substitution at position 36$)^{18}$ and $\mathrm{p} 66$ ShcQQ $(\mathrm{EE} \rightarrow \mathrm{QQ} \text { substitutions at positions } 132-133)^{19}$ were introduced into MEC cells by electroporation. A control line was generated using empty vector. Stably transfected cells were selected in medium containing $1 \mathrm{mg} / \mathrm{ml} \mathrm{G} 418$ (Gibco BRL, Life Technologies Italia srl, Milan). The Jurkat transfectants were previously described. ${ }^{18}$

Fresh peripheral blood CLL B cells were transiently cotransfected with $1 \mu \mathrm{g}$ GFP reporter/sample and $5 \mu \mathrm{g}$ empty vector (pcDNA3) or the same vector encoding p66Shc (purified using the EndoFree Plasmid Maxi kit from Qiagen Gmbh, Hilden, Germany) using the Human B-cell Nucleofector Kit (Amaxa Biosystems, Cologne, Germany). The transfection efficiency was consistently $40 \%$ or greater, as assessed by flow cytometric analysis of B cells transfected with the GFP reporter, and cell viability at the time of analysis (48 h post-transfection) was consistently $40-60 \%$. Surface S1P1 was measured on $\mathrm{GFP}^{+}$cells, gating on the population with the scatter parameters of live cells. RNA 
was extracted using the RNA mini kit (Qiagen Gmbh, Hilden, Germany) and retrotranscribed using the IScript cDNA synthesis kit (Biorad Laboratories Inc, Hercules, $\mathrm{CA})$.

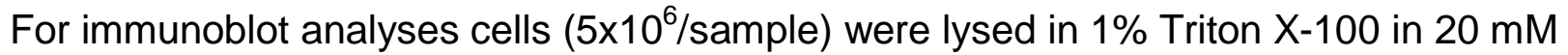
Tris- $\mathrm{HCl}(\mathrm{pH} 8), 150 \mathrm{mM} \mathrm{NaCl}$ (in the presence of a protease inhibitor cocktail). Immunoblot analysis of post-nuclear supernatants was carried out by chemiluminescence (SuperSignal® West Pico Chemiluminescent Substrate kit from Pierce, Rockford, IL) using as primary antibodies anti-ShcA (Upstate Biotechnology Inc, (Dundee, UK), anti-EDG1/S1P1 (Novus Biologicals, Cambridge, UK) or control antiactin (Millipore, Temecula, CA) antibodies and secondary peroxidase-labeled antibodies (Amersham Pharmacia Biotech, UK).

\section{RNA purification and Quantitative RT-PCR}

Total RNA was extracted from B cells from healthy donors, CLL patients, mice or MEC cells and retrotranscribed as described. ${ }^{20}$ Two independent reverse transcription reactions were performed on each RNA sample. Real-time PCR was performed in triplicate on each cDNA on 96-well optical PCR plates (Sarstedt AG, Nümbrecht, Germany) using SSo Fast ${ }^{\mathrm{TM}}$ EvaGreen ${ }^{\mathrm{R}}$ SuperMix (Biorad Laboratories Inc, Hercules, CA) according to the manufacturer's instructions and a CFX96 Real-Time system (BioRad Laboratories, Waltham, MA). After an initial denaturation for 3 min at $95^{\circ} \mathrm{C}$, denaturation in the subsequent 42 cycles was performed for $10 \mathrm{~s}$ at $95^{\circ} \mathrm{C}$, followed by $30 \mathrm{~s}$ primer annealing at $60^{\circ} \mathrm{C}$. Results were processed and analyzed using Bio-Rad CFX Manager version 1.5 software (Bio-Rad, Waltham, MA). Transcript levels were 
normalized to GAPDH, used as housekeeping gene. The primers used to amplify the cDNA fragments corresponding to human and mouse transcripts are listed in Table S1.

\section{Flow cytometry}

Cells were resuspended at $2 \times 10^{6} / \mathrm{ml}$ in serum-free medium added with $0.5 \%$ fatty acidfree BSA (Sigma-Aldrich srl, Milan, Italy) and incubated for 30 min at $37^{\circ} \mathrm{C}$ to allow recycling of intracellular S1P receptors to the cell surface. As a specificity control for S1P1 a sample was incubated with 100 nM FTY720 (Santa Cruz Biotechnology Inc, Santa Cruz, $\mathrm{CA}$ ) in the same medium for $30 \mathrm{~min}$ at $37^{\circ} \mathrm{C}$ to induce receptor downregulation. Cells were then incubated with anti-human EDG1/S1P1 (Novus Biologicals, Cambridge, UK), or anti-human EDG5/S1P2 (R\&D Systems, Minneapolis, MN), or antimouse EDG1/S1P1 (kindly provided by Jason Cyster) primary antibodies and Alexa Fluor® 488 Goat Anti-Mouse IgG or Alexa Fluor ${ }^{\circledR} 488$ Goat Anti-Rat IgG (Invitrogen, Eugene, OR) secondary antibodies. For the mouse experiments the analysis was carried out on gated $\mathrm{CD} 22^{+}$cells.

For B cell counts, peripheral blood and cell suspensions from lymph nodes, spleen and bone marrow were stained with fluorochrome-conjugated anti-CD3 and anti-CD22 antibodies (Becton Dickinson Bioscience, San Jose CA).

Flow cytometry was carried out using a FACScan flow cytometer (Becton Dickinson Bioscience). Data were analyzed and plotted using Flowjo (Tree Star Inc, Ashland, OR). 


\section{Cell treatments and ROS detection}

Cells were incubated with $400 \mu \mathrm{M} \mathrm{H}_{2} \mathrm{O}_{2}$ (Sigma-Aldrich srl) or $0.2 \mathrm{mM}$ Trolox (Merck Biosciences $\mathrm{GmbH}$, Schwalbach, Germany), respectively. After $3 \mathrm{~h}$ at $37^{\circ} \mathrm{C}$, cells were labeled for $30 \mathrm{~min}$ at $37^{\circ} \mathrm{C}$ with $5 \mu \mathrm{M} \mathrm{CM}-\mathrm{H}_{2}$ DCFDA (Molecular Probes, Invitrogen, Eugene, OR) and an aliquot was used to measure intracellular ROS by flow cytometry. After $24 \mathrm{~h}$ RNA was extracted and processed for qRT-PCR. Surface S1P1 was moreover measured by flow cytometry.

\section{Chemotaxis assays}

Chemotaxis assays were carried out using 24-well Transwell chambers with $5-\mu \mathrm{m}$ pore size polycarbonate membranes (Corning Life Sciences, Schiphol-Rijk, The Netherlands) essentially as described. ${ }^{21}$ Filters were soaked overnight in $0.5 \%$ fatty acid-free BSA (Sigma-Aldrich srl) in RPMI1640. The chemotaxis medium (500 $\mu$ l serum-free and 0.5\% fatty acid-free BSA medium) with or without $100 \mathrm{nM} \mathrm{S1P} \mathrm{(Sigma-Aldrich} \mathrm{srl)} \mathrm{was} \mathrm{placed}$ in the lower chamber, and $100 \mu \mathrm{l}$ cell suspension $\left(5 \times 10^{5}\right.$ cells/sample) in chemotaxis medium was placed in the upper chamber. After $3 \mathrm{~h}$ incubation at $37^{\circ} \mathrm{C}$ in humidified air with $5 \% \mathrm{CO}_{2}$, the upper chamber was emptied, filters were removed, and the cells in the lower chamber were counted by flow cytometry. The migration index was calculated by determining the ratio of migrated cells in treated versus untreated samples.

\section{Statistical analyses}

Mean values, standard deviation values and Student's $t$ test (unpaired) were calculated using the Microsoft Excel application. The difference between two consecutive samples 
From bloodjournal.hematologylibrary.org at HEALTH SERVICES LIB on October 18, 2012. For personal use only.

within the same population was determined by the Mann-Whitney Rank Sum test if the population was not normally distributed. A level of $p<0.05$ was considered statistically significant. 


\section{RESULTS}

\section{Defective S1P1 and S1P2 expression in CLL patients with unfavourable prognosis}

S1P1 expression in peripheral blood (PB) B cells was measured on a cohort of $78 \mathrm{CLL}$ patients divided, according to the IGHV mutational status, into two subsets of $32 \mathrm{M}-\mathrm{CLL}$ and $46 \mathrm{U}$-CLL patients. Since the levels of surface S1P1 undergo rapid changes depending on the local S1P concentration, ${ }^{12}$ we first measured the relative abundance of S1P1-specific transcripts in RNA from CLL B cells. B cells purified from PB lymphocytes from 13 healthy donors were used as controls. Patients with mutated IGHV (M-CLL) showed a large variability in the levels of S1P1 mRNA, as assessed by quantitative RT-PCR, with differences that were not statistically significant compared to healthy controls. Conversely, expression of S1P1 was markedly reduced in B cells from patients with unmutated IGHV (U-CLL) as compared to M-CLL and normal B cells (Fig. 1A, Fig.S1).

To confirm these results surface S1P1 was quantitated by flow cytometry. Cells were incubated for 30 min in serum-free medium in the presence of lipid-free BSA before adding the antibody to allow recycling of receptors that had been internalized in the presence of the high S1P concentrations in the blood. ${ }^{12,22}$ The pharmacological agonist, FTY720, which when phosphorylated by the endogenous S1P kinases binds to all S1P receptors with the exception of S1P2, inducing their rapid downregulation, ${ }^{23}$ was used as control. Consistent with the qRT-PCR results, no statistically significant differences 
were observed between M-CLL and healthy control B cells. Conversely, the levels of surface S1P1 were significantly reduced in U-CLL B cells when compared to M-CLL and normal B cells (Fig.1B). Hence S1P1 expression is selectively impaired in the U-CLL subset.

Histological analysis of lymph node (LN) and bone marrow (BM) from CLL patients has revealed that CLL cells associate with $\mathrm{CD} 40 \mathrm{~L}^{+} \mathrm{T}$ helper cells and follicular dendritic cells in GC-like structures, known as pseudo-GCs, where they are believed to receive not only survival but also proliferation signals. ${ }^{10}$ Since S1P2 has been recently implicated in the migration and survival of GC B cells, ${ }^{24}$ we measured the levels of S1P2 mRNA in normal and CLL B cells. Expression of S1P2 was profoundly impaired in CLL B cells when compared to normal B cells, independently of the IGHV mutational status, with UCLL cells displaying lower levels than M-CLL cells, as assessed both by qRT-PCR (Fig.1C, Fig.S1). and by flow cytometry (Fig.1D). This suggests that the behaviour of UCLL B cells during their residency within pseudo-GCs may be selectively affected by their defect in S1P2 expression.

\section{p66Shc controls S1P1 expression in B cells}

At variance with T cells, ${ }^{25}$ the factors that control S1P1 and S1P2 transcription in B cells have as yet not been identified. We have previously reported that the pro-oxidant ShcA isoform, p66Shc, ${ }^{26}$ acts as a negative regulator of proliferative and survival signals triggered by the BCR, and that p66Shc expression is impaired in CLL B cells, with the lowest levels found in U-CLL patients. ${ }^{20}$ This impairment in p66Shc expression was 
found to be causal to the intrinsic apoptosis defects of CLL B cells and resulted from the ability of this adaptor to modulate the Bcl-2 family genes, promoting the expression of proapoptotic members while inhibiting expression of antiapoptotic members ${ }^{20}$ Oxidants are known to profoundly affect gene transcription, ${ }^{27}$ suggesting that p66Shc could control the expression of other genes besides $\mathrm{Bcl}-2$ family members through its prooxidant activity, including S1P1 and S1P2.

To test this hypothesis we first measured S1P1 and S1P2 expression in MEC cells, a CLL-derived human B-cell line lacking p66Shc, stably transfected with either an expression vector encoding p66Shc, or empty vector. p66Shc-expressing MEC cells displayed significantly higher levels of both S1P1 mRNA and surface S1P1 as compared to the control transfectant (Fig.2A,B). Conversely, p66Shc did not increase S1P2 expression above the very low levels detected in MEC cells (Fig.2C,D). Consistent with the S1P1 defect, the chemotactic response of p66Shc-expressing cells towards S1P was more robust compared to control cells (Fig.2E).

To further investigate the implication of p66Shc in S1P1 expression we used splenic B cells from wild-type and p66Shc ${ }^{--}$mice. Cells were purified by immunomagnetic sorting and S1P1 as well as S1P2 expression was measured by qRT-PCR and flow cytometry. In agreement with the results obtained on the MEC transfectants, $\mathrm{p} 66 \mathrm{Shc}^{-/} \mathrm{B}$ cells were found to be defective in S1P1 but not S1P2 expression (Fig.3A,B, Fig.S2A). This defect was observed in all peripheral lymphoid tissues analyzed, including spleen, LN and PB (Fig.3C, Fig.S2C). Of note, the variability in the S1P1 levels in B cells from wild-type 
mice correlated with the levels of p66Shc (Fig.S2B), further supporting the implication of p66Shc in the control of S1P1 expression. Interestingly, p66Shc deficiency did not affect S1P1 expression in T cells, as assessed both in Jurkat T cells stably transfected with either empty vector or the p66Shc-encoding construct, and in splenic T cells from wildtype and p66Shc ${ }^{-/-}$mice (Fig.S3A,B). Consistent with the S1P1 defect, migration towards S1P was severely impaired in p66Shc ${ }^{-1}$ B cells compared to their wild-type counterparts (Fig.3D). Moreover p66Shc ${ }^{-/-}$mice showed a reduction in B-cell (but not $\mathrm{T}$ cell) counts in PB, paralleled by an increase in LN, spleen and BM (Fig.S3C, Fig.S4).

The finding that p66Shc controls S1P1 expression suggests that the S1P1 defect observed in U-CLL B cells could be caused by their deficiency in p66Shc. To address this issue B cells purified from M-CLL and U-CLL patients were nucleofected with the p66Shc-encoding construct and the levels of S1P1 were measured by qRT-PCR. Reconstitution of p66Shc expression in CLL B cells resulted in a significant increase in the levels of S1P1 mRNA, which was confirmed by flow cytometry (Fig.4A,B). At variance, p66Shc did not affect S1P2 expression (data not shown). Hence the defect in S1P1 expression in B cells from U-CLL patients is caused, at least in part, by the impairment in p66Shc expression in these cells.

\section{Modulation of S1P1 expression by p66Shc is mediated by its pro-oxidant activity}

The pro-oxidant activity of p66Shc is mediated by two different mechanisms reflecting its dual intracellular localization, i.e. cytosolic and mitochondrial. The cytosolic pool 
enhances both the homoeostatic and stress-dependent ROS production by inhibiting the expression of ROS-scavenging enzymes through its ability to promote cytosolic translocation of FoxO transcription factors. This activity is dependent on phosphorylation of S36 in the N-terminal collagen homology domain $(\mathrm{CH} 2){ }^{28}$ The mitochondrial pool, which is replenished from the cytosolic pool under conditions of stress, ${ }^{29}$ increases ROS production by binding cytochrome-c, thereby interrupting the respiratory chain. ${ }^{19}$ This activity requires residues $\mathrm{E} 132 \mathrm{Q}-\mathrm{E} 133 \mathrm{Q}$ within the cytochromec binding domain, which spans a short region adjacent to the $\mathrm{CH} 2$ domain (Fig.5A).

To address the role of the pro-oxidant function of p66Shc in the regulation of S1P1 expression we generated stable MEC transfectants expressing either a mutant carrying a $S \rightarrow A$ substitution at position 36 (p66ShcSA) or a mutant carrying a double $E \rightarrow Q$ substitution at positions 132-133 (p66ShcQQ) (Fig.5A). The empty vector transfectant lacking p66Shc and the transfectant expressing the wild-type protein were used as controls. We first measured the homeostatic ROS production in all transfectants by flow cytometric analysis of cells loaded with $\mathrm{CM}-\mathrm{H}_{2}$ DCFDA, a cell-permeant probe that becomes fluorescent following oxidation. The relative levels of ROS were found to be significantly higher in p66Shc-expressing MEC cells when compared to controls. Conversely, no difference was observed in the presence of either p66ShcSA or p66ShcQQ (Fig.5B).

Expression of S1P1 was next measured in all transfectants both by qRT-PCR and by flow cytometry. At variance with wild-type p66Shc, the levels of S1P1 mRNA (Fig.S5A) 
and surface receptor (Fig.5C) were comparable between MEC cells transfected with empty vector and the MEC transfectants expressing either the p66ShcSA or the p66ShcQQ mutant. No effect was observed on S1P2 expression (data not shown). Consistent with these findings, the chemotactic response to S1P of MEC transfectants expressing the p66Shc mutants was comparable to the empty vector transfectant (Fig.S5B). Collectively, these data suggest that the ability of p66Shc to modulate S1P1 expression is mediated by its ROS-elevating activity. To further assess this possibility the control MEC transfectant lacking p66Shc was treated for $24 \mathrm{~h}$ with $\mathrm{H}_{2} \mathrm{O}_{2}$. Conversely, the p66Shc-expressing transfectant was treated for the same time with Trolox, a cell-permeable vitamin E analogue with strong anti-oxidant activity. S1P1 expression correlated with the redox state of the cell, increasing in the presence of high levels of oxidants (Fig.5D, Fig.S5C). Moreover the increase in S1P1 expression induced by p66Shc was partly abrogated by Trolox (Fig.5E, Fig.S5D), further strengthening the notion that p66Shc controls S1P1 expression by promoting ROS production. Of note, consistent with these findings, homeostatic ROS production in CLL cells was decreased when compared to normal B cells, with a statistically significant difference between MCLL and U-CLL B cells (Fig.S6).

\section{CCR7 expression is regulated by p66Shc}

Expression of the homing receptor CCR7, which responds to the chemokine CCL21 that is present at high levels in peripheral lymphoid organs, follows an opposite trend compared to S1P1, decreasing as the result of ligand-dependent internalization to allow 
trafficking to proceed in the absence of antigen recognition. In addition, CCR7 expression is shut off at the transcriptional level in activated lymphocytes that have undergone several divisions, with a concomitant increase in S1P1 expression, which allows the egress signal delivered by S1P1 to overcome the CCR7-mediated retention signal. ${ }^{30}$ CCR7 expression is enhanced in CLL B cells (see also Fig.6A), with a greater increase in the presence of lymphoadenopathy. ${ }^{31}$

We addressed the potential role of p66Shc in the control of CCR7 expression, using both the MEC B-cell transfectants and p66Shc ${ }^{-/-}$B cells. Quantitative RT-PCR analysis showed a tight inverse correlation between p66Shc and CCR7 expression (Fig.6B,C; Fig.S2A). Moreover, reconstitution of p66Shc in CLL B cells resulted in a reduction in CCR7 mRNA (Fig.6D), indicating that the p66Shc defect in CLL B cells is responsible at least in part for their upregulation of CCR7 expression.

To understand whether the impairment in CCR7 expression in the presence of p66Shc is regulated by the same mechanism as S1P1 we measured the levels of CCR7 mRNA in the MEC transfectants expressing the p66Shc mutants devoid of ROS-elevating activity. CCR7 was found to be expressed at levels comparable to control cells in the MEC transfectants expressing either the p66ShcSA or the p66ShcQQ mutant (Fig.6B). Hence the genes encoding CCR7 and S1P1 are controlled in opposite directions by the ROS-elevating activity of p66Shc. 


\section{DISCUSSION}

The levels of S1P receptors are tightly regulated during lymphocyte trafficking, thereby setting their dwell time in peripheral lymphoid organs. S1P receptors are rapidly downregulated in the S1P-rich circulatory fluids by ligand-dependent internalization. They are then effectively recycled to the cell surface in the S1P-poor lymphoid organs, becoming resensitized to promote lymphocyte egress. ${ }^{22}$ Modulation of the levels of S1P receptors by local S1P pools also controls the location and movements of lymphocytes within specific regions of peripheral lymphoid organs. ${ }^{12,24}$ Additionally, the levels of S1P receptors are regulated by de novo gene transcription, as shown for S1P1 in activated T cells that must leave the lymphoid organs to reach the sites of infection where their effector function is required. ${ }^{15,32}$

Among the S1P receptors S1P1 plays a crucial role in regulating the egress of $\mathrm{T}$ and $\mathrm{B}$ cells from peripheral lymphoid organs towards blood and lymph, overcoming the retention signals provided by CCR $7 .{ }^{30}$ Our data highlight a defect in S1P1 expression in U-CLL B cells and confirm a previous report indicating that CLL B cells overexpress CCR7, which results in enhanced transendothelial migration towards CCL19 and CCL21. ${ }^{31}$ We hypothesize that the impairment in S1P1 expression in U-CLL B cells will result in an abnormal balance between retention (CCR7-mediated) and egress (S1P1mediated) signals that may increase their dwell time in peripheral lymphoid organs. In this respect, it is noteworthy that the levels of CCR7 were found to be significantly higher in CLL patients with clinical lymphoadenopathy as compared to patients lacking 
this alteration. ${ }^{31}$ Moreover, although no stastically significant difference in S1P1 expression was observed between M-CLL and control B cells, M-CLL patients with the lowest levels of S1P1 had a higher number of lymph nodes involved when compared to high expressers (Trentin, unpublished observations).

CLL B cells overexpress chemokine receptors, such as CXCR4 and CXCR5, the ligands of which are produced at supraphysiological concentrations in the stromal microenvironment in these patients ${ }^{5,33}$ and provide survival signals that contribute to the prolonged lifespan of CLL B cells. ${ }^{34}$ An extended dwell time in peripheral lymphoid tissues is expected to favour these interactions. Moreover a sustained interaction of the signaling-competent BCRs on U-CLL B cells ${ }^{9}$ with low-affinity self-antigens is expected to result in productive signaling, accounting for the presence of proliferating cells in these tissues. ${ }^{1}$ This scenario is supported by the results of clinical trials with the Btk inhibitor PCl-32765, showing a sustained regression of lymphoadenopathy accompanied by transient lymphocytosis, ${ }^{35}$ which was also observed in a mouse model of CLL. ${ }^{36}$ The notion of the potential importance of an extended dwell time in a niche favourable to cell survival and proliferation is in line with the finding that CCR7 regulates E $\mu$-Myc lymphoma homing to $L N$ and spleen, providing the neoplastic CCR7 ${ }^{\text {high }}$ cells with a survival advantage.$^{37}$ Interestingly, p66Shc ${ }^{-/-}$mice display a moderate B cell lymphopenia, which correlates with their S1P1 expression defect in B cells. The residual levels of S1P1 in p66Shc ${ }^{-/}$cells are likely to be sufficient to delay B-cell egress from the lymphoid organs in these mice. Together with the enhanced BCR signaling in p66Shc ${ }^{-/-}$ $\mathrm{B}$ cells, ${ }^{20}$ an extended dwell time of these cells in the specialized niche of the lymphoid 
microenvironment may account for the spontaneous GC formation observed in these mice. ${ }^{5}$ Although p66Shc ${ }^{-/}$mice do not develop lymphoid neoplasias, this might provide a selective advantage to $B$ cells that have acquired oncogenic mutations, as proposed for the priming effect of S1P2 deletion on human diffuse large B-cell lymphoma (DLBCL) development. ${ }^{38}$

The outcome of the defect in S1P2 expression in both M-CLL and U-CLL B cells appears more elusive. S1P2 inhibits the response of GC B cells to follicular chemoattractants in the T-cell zone, thereby favouring their confinement to the S1P-low niche in the follicle center. S1P2 inhibits moreover Akt activation, thereby restricting GC B cell survival. ${ }^{24}$ The B cell-specific S1P2 deletion in the mouse results in the development of a lymphoma with the features typical of human DLBCL, which is preceded by spontaneous GC formation. ${ }^{38}$ CLL B cells have been shown to form pseudo-GCs both in peripheral lymphoid tissues and BM. ${ }^{10}$ Although these structures do not have the ordered architecture of true GCs, the defect in S1P2 expression might not only directly contribute to extending their survival in the presence of S1P but also increase their opportunities to interact with other cells that have been implicated in delivering pro-survival and/or proliferation cues within pseudo-GC, including T cells (CD40L), FDC (CD44), nurse-like cells (CXCL12, CXCL13, BAFF) and bone marrow stromal cells (CXCL12). ${ }^{5}$

The alterations in both S1P1 and CCR7 expression in CLL B cells appear to be causally related to the concomitant impairment in p66Shc expression, as they could be 
corrected, at least in part, by increasing the levels of p66Shc. This link is further supported by the results obtained on the p66Shc B-cell transfectants as well as on B cells from p66Shc ${ }^{-/}$mice. Conversely, p66Shc deficiency does not affect S1P1 expression in T cells. Hence p66Shc emerges in B cells as a new player in the coordinate regulation of these two key regulators of lymphocyte trafficking. At variance with other immune cell types, the factors controlling expression of S1P1 and CCR7 expression in B cells are as yet largely unknown. The data presented in this report identify S1P1 and CCR7 as ROS-responsive genes in B cells and provide evidence that p66Shc participates in the control of their expression through its pro-oxidant activity, as shown both genetically, using mutants lacking the ability to promote ROS accumulation, and by pharmacological modulation of intracellular ROS. In this context, it is noteworthy that quantification of intracellular ROS in B cells from patients who participated in this study showed that CLL B cells had lower levels of homeostatic ROS when compared to normal B cells. This finding appears at variance with a previous report documenting higher ROS levels in CLL B cells. ${ }^{39}$ Nevertheless, the authors showed that the levels of ROS correlated with treatment, with levels in untreated patients actually lower than in normal B cells, ${ }^{39}$ albeit not to the same extent as in our samples. Although our study will need to be extended to a larger number of patients as well as healthy controls, the fact that we limited our analysis to untreated patients might account for the apparent discrepancy. An intriguing observation is that, at variance with CCR7, which is overexpressed both in M-CLL and U-CLL B cells, with the highest levels in the latter, the defect in S1P1 can be consistently observed only in U-CLL, notwithstanding the fact that p66Shc expression and ROS production are impaired in M-CLL, albeit to a lesser 
extent than in U-CLL. The results suggest that $C C R 7$ may more sensitive to the prooxidant activity of p66Shc when compared to S1P1.

Despite the recent advances in the characterization of B-CLL, this neoplasia remains incurable. The issue is further confounded by the highly variable clinical course of these patients, with a heterogeneous pattern of clinical features ranging from indolent to aggressive conditions. For this reason the identification of markers that might predict an unfavourable outcome remains a priority. A number of predictive markers have been proposed, some of which are used to characterize discrete CLL subgroups, including cytogenetic abnormalities, the IGHV mutational status, ectopic ZAP-70 expression and surface CD38. ${ }^{3,40}$ Our data provide evidence of a selective impairment in S1P1 and S1P2 expression in leukemic cells from patients classified as with unfavourable prognosis based on the lack of IGHV mutations, which suggests the interesting possibility that S1P1 and S1P2 deficiency could be used as new markers for predicting disease behaviour. Prospective studies on larger cohorts of patients will be required to assess this possibility. 
Acknowledgements

The authors wish to thank John L Telford and Paolo Bernardi for productive discussions, Jason Cyster for his generous gift of anti-mouse S1P1 and for useful suggestions and Sonia Grassini for technical assistance. This work was generously supported by the Italian Association for Cancer Research (AIRC). The support of FIRB, ITT-Regione Toscana and Fondazione CaRiParo and CaRiVerona is also acknowledged. NC is supported by a FIRC fellowship.

Authorship contributions and disclosure of conflicts of interest. NC, LP, LT, GS and CTB designed research and analyzed and interpreted data; NC, LP, OML and EC performed research; FF, CG, PGP, EM and FFo contributed vital reagents; NC, LP, LT, GS and CTB drafted the manuscript. There is no conflict of interest. 


\section{REFERENCES}

1. Caligaris-Cappio F, Ghia P. Novel insights in chronic lymphocytic leukemia: are we getting closer to understanding the pathogenesis of the disease? J Clin Oncol. 2008;26(27):4497-4503.

2. Klein U, Dalla-Favera R. New insights into the pathogenesis of chronic lymphocytic leukemia. Semin Cancer Biol. 2010;20(6):377-383.

3. Stevenson FK, Krysov S, Davies AJ, Steele AJ,Packham G. B-cell receptor signaling in chronic lymphocytic leukemia. Blood. 2011;118(16):4313-4320.

4. Packham G, Stevenson FK. Bodyguards and assassins: Bcl-2 family proteins and apoptosis control in chronic lymphocytic leukaemia. Immunology. 2005;114(4):441-449.

5. Burger JA. Nurture versus nature: the microenvironment in chronic lymphocytic leukemia. Hematology Am Soc Hematol Educ Program. 2011;2011:96-103.

6. Kehrl JH, Hwang IY, Park C. Chemoattract receptor signaling and its role in lymphocyte motility and trafficking. Curr Top Microbiol Immunol. 2009;334:107127.

7. Lopez-Giral S, Quintana NE, Cabrerizo M, et al. Chemokine receptors that mediate B cell homing to secondary lymphoid tissues are highly expressed in B cell chronic lymphocytic leukemia and non-Hodgkin lymphomas with widespread nodular dissemination. J Leukoc Biol. 2004;76(2):462-471. 
8. Mockridge $\mathrm{Cl}$, Potter KN, Wheatley I, et al. Reversible anergy of slgM-mediated signaling in the two subsets of CLL defined by VH-gene mutational status. Blood. 2007;109(10):4424-4431.

9. Petlickovski A, Laurenti L, Li X, et al. Sustained signaling through the B-cell receptor induces $\mathrm{Mcl}-1$ and promotes survival of chronic lymphocytic leukemia B cells. Blood. 2005;105(12):4820-4827.

10. Ghia P, Chiorazzi N, Stamatopoulos K. Microenvironmental influences in chronic lymphocytic leukaemia: the role of antigen stimulation. $J$ Intern Med. 2008;264(6):549-562.

11. Chu CC, Catera R, Zhang L, et al. Many chronic lymphocytic leukemia antibodies recognize apoptotic cells with exposed nonmuscle myosin heavy chain IIA: implications for patient outcome and cell of origin. Blood. 2010;115(19):39073915.

12. Cyster JG, Schwab SR. Sphingosine-1-Phosphate and Lymphocyte Egress from Lymphoid Organs. Annu Rev Immunol. 2012;30:69-94.

13. Pappu R, Schwab SR, Cornelissen I, et al. Promotion of lymphocyte egress into blood and lymph by distinct sources of sphingosine-1-phosphate. Science. 2007;316(5822):295-298.

14. Le Stunff H, Milstien S, Spiegel S. Generation and metabolism of bioactive sphingosine-1-phosphate. J Cell Biochem. 2004;92(5):882-899.

15. Matloubian M, Lo CG, Cinamon G, et al. Lymphocyte egress from thymus and peripheral lymphoid organs is dependent on S1P receptor 1. Nature. 2004;427(6972):355-360. 
16. Migliaccio E, Giorgio M, Mele S, et al. The p66shc adaptor protein controls oxidative stress response and life span in mammals. Nature. 1999;402(6759):309-313.

17. Stacchini A, Aragno M, Vallario A, et al. MEC1 and MEC2: two new cell lines derived from B-chronic lymphocytic leukaemia in prolymphocytoid transformation. Leuk Res. 1999;23(2):127-136.

18. Pacini S, Pellegrini M, Migliaccio E, et al. p66SHC promotes apoptosis and antagonizes mitogenic signaling in T cells. Mol Cell Biol. 2004;24(4):1747-1757.

19. Giorgio M, Migliaccio E, Orsini F, et al. Electron transfer between cytochrome c and p66Shc generates reactive oxygen species that trigger mitochondrial apoptosis. Cell. 2005;122(2):221-233.

20. Capitani N, Lucherini OM, Sozzi E, et al. Impaired expression of p66Shc, a novel regulator of B-cell survival, in chronic lymphocytic leukemia. Blood. 2010;115(18):3726-3736.

21. Patrussi L, Ulivieri C, Lucherini OM, et al. p52Shc is required for CXCR4dependent signaling and chemotaxis in T cells. Blood. 2007;110(6):1730-1738.

22. Lo CG, Xu Y, Proia RL, Cyster JG. Cyclical modulation of sphingosine-1phosphate receptor 1 surface expression during lymphocyte recirculation and relationship to lymphoid organ transit. J Exp Med. 2005;201(2):291-301.

23. Graler MH,Goetzl EJ. The immunosuppressant FTY720 down-regulates sphingosine 1-phosphate G-protein-coupled receptors. FASEB J. 2004;18(3):551-553. 
24. Green JA, Suzuki K, Cho B, et al. The sphingosine 1-phosphate receptor S1P maintains the homeostasis of germinal center B cells and promotes niche confinement. Nat Immunol. 2011;12(7):672-680.

25. Carlson CM, Endrizzi BT, Wu J, et al. Kruppel-like factor 2 regulates thymocyte and T-cell migration. Nature. 2006;442(7100):299-302.

26. Finetti F, Savino MT, Baldari CT. Positive and negative regulation of antigen receptor signaling by the Shc family of protein adapters. Immunol Rev. 2009;232(1):115-134.

27. Huang H, Tindall DJ. Dynamic FoxO transcription factors. J Cell Sci. 2007;120(Pt 15):2479-2487.

28. Nemoto S, Finkel T. Redox regulation of forkhead proteins through a p66shcdependent signaling pathway. Science. 2002;295(5564):2450-2452.

29. Pinton $\mathrm{P}$, Rimessi $\mathrm{A}$, Marchi $\mathrm{S}$, et al. Protein kinase $\mathrm{C}$ beta and prolyl isomerase 1 regulate mitochondrial effects of the life-span determinant p66Shc. Science. 2007;315(5812):659-663.

30. Pham TH, Okada T, Matloubian M, Lo CG, Cyster JG. S1P1 receptor signaling overrides retention mediated by $\mathrm{G}$ alpha i-coupled receptors to promote $\mathrm{T}$ cell egress. Immunity. 2008;28(1):122-133.

31. Till KJ, Lin K, Zuzel M, Cawley JC. The chemokine receptor CCR7 and alpha4 integrin are important for migration of chronic lymphocytic leukemia cells into lymph nodes. Blood. 2002;99(8):2977-2984. 
32. Bai A, Hu H, Yeung M, Chen J. Kruppel-like factor 2 controls T cell trafficking by activating L-selectin (CD62L) and sphingosine-1-phosphate receptor 1 transcription. J Immunol. 2007;178(12):7632-7639.

33. Trentin L, Cabrelle A, Facco M, et al. Homeostatic chemokines drive migration of malignant B cells in patients with non-Hodgkin lymphomas. Blood. 2004;104(2):502-508

34. Munk Pedersen I, Reed J. Microenvironmental interactions and survival of CLL B-cells. Leuk Lymphoma. 2004;45(12):2365-2372.

35. Byrd JC, Blum KA, Burger JA, et al. Activity and tolerability of the Bruton's tyrosine kinase (Btk) inhibitor PCI-32765 in patients with chronic lymphocytic leukemia/small lymphocytic lymphoma (CLL/SLL): interim results of a phase lb/II study. J Clin Oncol (ASCO Annual Meeting Proceedings). 2011;29(15):6508.

36. de Rooij MF, Kuil A, Geest CR, et al. The clinically active BTK inhibitor PCI32765 targets B-cell receptor- and chemokine-controlled adhesion and migration in chronic lymphocytic leukemia. Blood. 2012;119(11):2590-2594.

37. Rehm A, Mensen A, Schradi K, et al. Cooperative function of CCR7 and lymphotoxin in the formation of a lymphoma-permissive niche within murine secondary lymphoid organs. Blood. 2011;118(4):1020-1033.

38. Cattoretti G, Mandelbaum J, Lee N, et al. Targeted disruption of the S1P2 sphingosine 1-phosphate receptor gene leads to diffuse large B-cell lymphoma formation. Cancer Res. 2009;69(22):8686-8692.

39. Tomic J, Lichty B, Spaner DE. Aberrant interferon-signaling is associated with aggressive chronic lymphocytic leukemia. Blood. 2011;117(9):2668-2680. 
From bloodjournal.hematologylibrary.org at HEALTH SERVICES LIB on October 18, 2012. For personal use only.

40. Dal-Bo M, Bertoni F, Forconi F, et al. Intrinsic and extrinsic factors influencing the clinical course of B-cell chronic lymphocytic leukemia: prognostic markers with pathogenetic relevance. J Trans/ Med. 2009;7:76. 


\section{FIGURE LEGENDS}

Figure 1. Impaired S1P1 and S1P2 expression in CLL B cells from patients with unmutated IGHV. A,C. Quantitative RT-PCR analysis of S1P1 (A) or S1P2 (C) mRNA in purified peripheral B cells from either healthy donors ( $\mathrm{ctr} ; \mathrm{n}=13$ ) or CLL patients with mutated $(\mathrm{M} ; \mathrm{n}=28$ for S1P1; $\mathrm{n}=25$ for S1P2) or unmutated $(\mathrm{U} ; \mathrm{n}=42$ for S1P1; $\mathrm{n}=36$ for S1P2) IGHV. The relative abundance of gene transcripts was determined on triplicate samples using the ddCt method and is expressed as normalized fold expression (mean +/- SD). B,D. Flow cytometric analysis of surface S1P1 (B) or S1P2 (D) on purified peripheral $B$ cells from either healthy donors (ctr; $n=10$ for S1P1; $n=11$ for S1P2) or CLL patients with mutated $(M ; n=18$ for S1P1; $n=16$ for S1P2) or unmutated $(U ; n=18$ for $\mathrm{S} 1 \mathrm{P} 1 ; \mathrm{n}=16$ for S1P2) IGHV. The data in the histograms are expressed as mean fluorescence intensity (MFI) +/- SD. Representative FACS profiles of S1P1 (B) or S1P2 (D) are shown. Specificity controls for each donor/patient included a sample incubated with secondary antibody alone ( $\alpha$-mouse) and, for S1P1 stainings, a sample preincubated with FTY720 to induce receptor downregulation. $p<0.001,{ }^{* * *} ; \mathrm{p}<0.01,{ }^{* *}$; $\mathrm{p}<0.05,{ }^{*}$ (Mann-Whitney Rank Sum test).

Figure 2. p66Shc controls S1P1 expression in B cells. A,C. Quantitative RT-PCR analysis of S1P1 (A) or S1P2 (C) mRNA in MEC B cells stably transfected with either empty vector (MEC ctr) or an expression construct encoding p66Shc (MEC p66). The relative abundance of gene transcripts was determined on triplicate samples from at least three independent experiments using the ddCt method and is expressed as 
normalized fold expression (mean +/- SD). B,D. Flow cytometric analysis of surface S1P1 (B) or S1P2 (D) on MEC B cells stably transfected with either empty vector (MEC ctr) or an expression construct encoding p66Shc (MEC p66). The data in the histograms are expressed as mean fluorescence intensity (MFI) +/- SD. Representative FACS profiles of S1P1 (B) or S1P2 (D) are shown. Specificity controls for each experiment included a sample incubated with secondary antibody alone ( $\alpha$-mouse) and, for S1P1 stainings, a sample preincubated with FTY720 to induce receptor downregulation. E. Migration of the control and p66Shc-expressing MEC transfectants, measured after treatment for $3 \mathrm{~h}$ with S1P (100 nM). The data, obtained on duplicate samples from at least 3 independent experiments, are presented as mean migration index $+/-$ SD (ratio migrated cells in S1P-treated samples vs untreated samples). $p<0.001,{ }^{* * *} ; p<0.01,{ }^{* *}$.

\section{Figure 3. Defective S1P1 expression in B cells from p66Shc ${ }^{-/-}$mice. A,B.}

Quantitative RT-PCR analysis of S1P1 (A) or S1P2 (B) mRNA in purified splenic B cells from wild-type $(+/+)$ or p66Shc ${ }^{-/}(-/-)$mice. The relative abundance of gene transcripts was determined on triplicate samples from at least 6 wild-type or $\mathrm{p} 66 \mathrm{Shc}^{-/-}$mice using the ddCt method and is expressed as normalized fold expression (mean +/- SD). C. Flow cytometric analysis of surface S1P1 on splenic, lymph node (LN) or peripheral blood (PB) B cells (analysis on gated $\mathrm{CD}_{2} 2^{+}$cells) from wild-type $(+/+)$or p66Shc ${ }^{-/-}(-/-)$ mice. The data are expressed as mean fluorescence intensity (MFI) +/- SD ( $n \geq 9$ mice/group). Specificity controls for each experiment included a sample incubated with secondary antibody alone and a sample preincubated with FTY720 to induce receptor downregulation. $p<0.001,{ }^{* \star *} ; p<0.01,{ }^{* *} ; p<0.05,{ }^{*}$ (Mann-Whitney Rank Sum test). D. 
Migration of purified splenic B cells from wild-type (+/+) or p66Shc ${ }^{-/-}(-/)$mice, measured after treatment for $3 \mathrm{~h}$ with S1P $(100 \mathrm{nM})$. The data, obtained on duplicate samples from at least 3 independent experiments, are presented as mean migration index + /- SD (ratio migrated cells in S1P-treated samples vs untreated samples). $\mathrm{p}<0.001,{ }^{* * *}$.

Figure 4. p66Shc reconstitution in CLL B cells results in enhanced S1P1 expression. Quantitative RT-PCR (A) and flow cytometric (B) analysis of S1P1 expression in peripheral blood $B$ cells purified from CLL patients with either mutated ( $M$; $\mathrm{n}=7)$ or unmutated $(\mathrm{U} ; \mathrm{n}=9) / G H V$ nucleofected with either empty vector (vector $\mathrm{ctr}$ ) or an expression construct encoding p66Shc (p66). The analysis was carried out $48 \mathrm{~h}$ post-transfection on $\mathrm{GFP}^{+}$live cells. All samples were checked for reconstitution of p66Shc expression by qRT-PCR (data not shown). An immunoblot analysis of p66Shc and S1P1 expression on a representative experiment is shown on the right. The relative abundance of gene transcripts was determined on triplicate samples from each patient using the ddCt method and is expressed as normalized fold expression (mean +/- SD; empty vector controls taken as 1 for all CLL samples). The data in the histograms showing the quantification of surface S1P1 are expressed as mean fluorescence intensity (MFI) of p66-transfected cells / MFI of vector ctr-transfected cells (normalized to 1 in all empty vector controls) $+/-$ SD. $p<0.001,{ }^{* * *} ; p<0.01,{ }^{* *} ; p<0.05,{ }^{*}$.

\section{Figure 5. p66Shc controls S1P1 expression through its pro-oxidant activity. A.} Immunoblot analysis of Shc expression in MEC B cells stably transfected with empty 
vector (MEC ctr) or an expression construct encoding either wild-type p66Shc (MEC p66) or the S36A (MEC p66SA) or EE132/133QQ (MEC p66QQ) mutants. A control anti-actin blot of the stripped filter is shown below. The migration of molecular mass markers is indicated. The domain structure of p66Shc highlighting the location of the aminoacid residues substituted in the mutants is schematized at the top of the panel. B. Flow cytometric analysis of ROS production in the MEC B-cell transfectants loaded with the ROS-sensitive probe, $\mathrm{CM}-\mathrm{H}_{2} \mathrm{DCFDA}$. The data are expressed as relative ROS production, using as a reference control (empty vector) MEC cells $(n=4)$. C. Flow cytometric analysis of surface S1P1 in the MEC B cell transfectants. The data are expressed as mean fluorescence intensity (MFI) $+/-S D(n=4)$. Specificity controls for each S1P1 staining experiment included a sample incubated with secondary antibody alone and a sample preincubated with FTY720 to induce receptor downregulation. D,E. Flow cytometric analysis of S1P1 mRNA in control MEC cells treated for $24 \mathrm{~h}$ with 400 $\mu \mathrm{M} \mathrm{H}_{2} \mathrm{O}_{2}(\mathrm{D})$ and in p66Shc-expressing MEC cells treated for $24 \mathrm{~h}$ with $0.2 \mathrm{mM}$ Trolox (E). Under these conditions a $3 \mathrm{~h}$ treatment with $\mathrm{H}_{2} \mathrm{O}_{2}$ increased intracellular ROS in control (empty vector) MEC by 2-fold, while Trolox reduced intracellular ROS in p66Shcexpressing MEC cells by $65 \% . p<0.001,{ }^{* * *} ; p<0.01,{ }^{* *} ; p<0.05,{ }^{*}$.

Figure 6. CCR7 expression is negatively regulated by p66Shc through its prooxidant activity. A. Quantitative RT-PCR analysis of CCR7 mRNA in purified peripheral B cells from either healthy donors ( $c t r ; n=8)$ or CLL patients with mutated ( $M$; $\mathrm{n}=29)$ or unmutated $(\mathrm{U} ; \mathrm{n}=28) I G H V$. The relative abundance of gene transcripts was determined on triplicate samples using the $\mathrm{ddCt}$ method and is expressed as 
normalized fold expression (mean +/- SD). $p<0.001,{ }^{* * *} ; p<0.01,{ }^{* *}$ (Mann-Whitney Rank Sum test). B. Quantitative RT-PCR analysis of CCR7 mRNA in MEC B cells stably transfected with empty vector (MEC ctr) or an expression construct encoding either wild-type p66Shc (MEC p66) or the S36A (MEC p66SA) or EE132/133QQ (MEC p66QQ) mutants. $p<0.01,{ }^{* *}$. C. Quantitative RT-PCR analysis of CCR7 mRNA in purified splenic B cells from wild-type $(+/+)$ or p66Shc ${ }^{-/}(-/-)$mice. The relative abundance of gene transcripts was determined on triplicate samples from at least 5 wild-type or p66Shc ${ }^{-/}$mice. $p<0.01,{ }^{* *}$ (Mann-Whitney Rank Sum test). D. Quantitative RT-PCR analysis of CCR7 expression in peripheral blood B cells purified from CLL patients with either mutated $(\mathrm{M} ; \mathrm{n}=7)$ or unmutated $(\mathrm{U} ; \mathrm{n}=6) I G H V$ nucleofected with either empty vector (vector ctr) or an expression construct encoding p66Shc (p66). The analysis was carried out $48 \mathrm{~h}$ post-transfection. All samples were checked for reconstitution of p66Shc expression by qRT-PCR (data not shown). The relative abundance of gene transcripts was determined on triplicate samples from each patient using the ddCt method and is expressed as normalized fold expression (mean +/- SD; empty vector controls taken as 1 for all CLL samples). $p<0.001,{ }^{* * *} ; p<0.05,{ }^{*}$. 
Figure 1

A
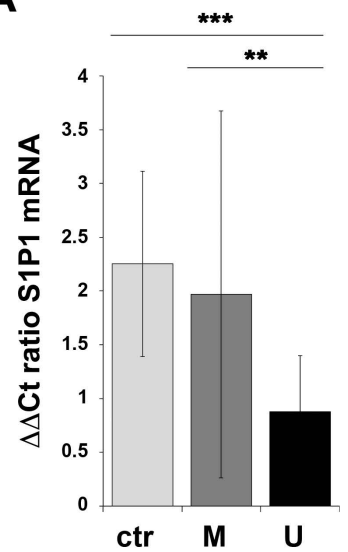

C

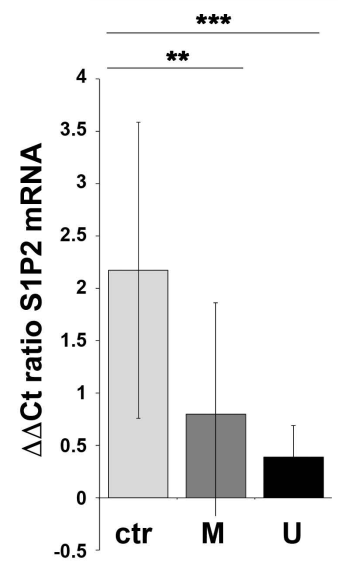

B
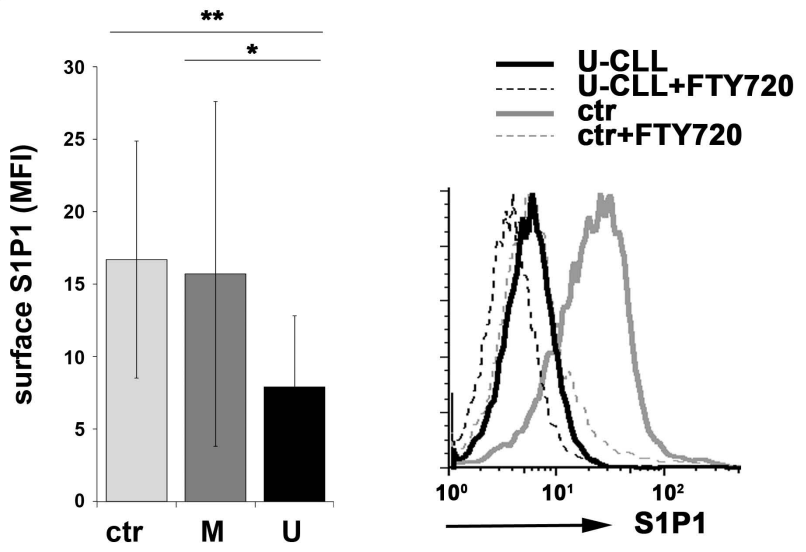

D

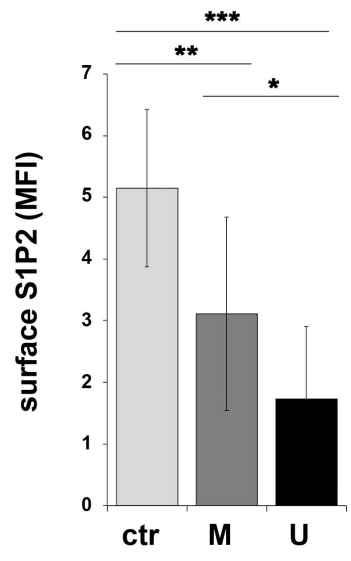

U-CLL U-CLL, $\alpha$-mouse ctr ctr, $\alpha$-mouse

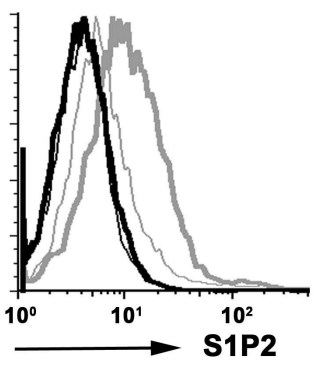


Figure 2

A

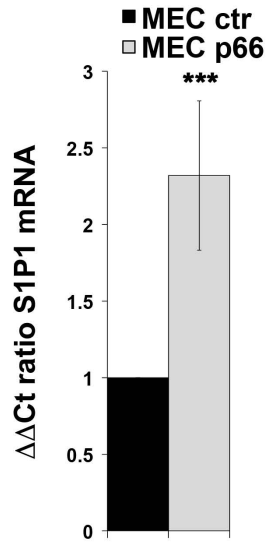

C

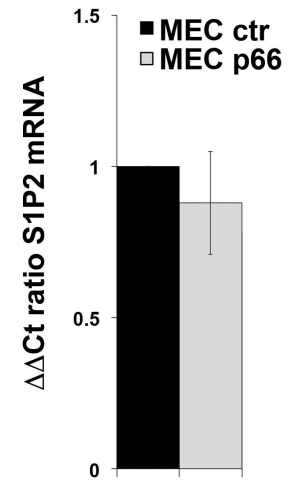

B
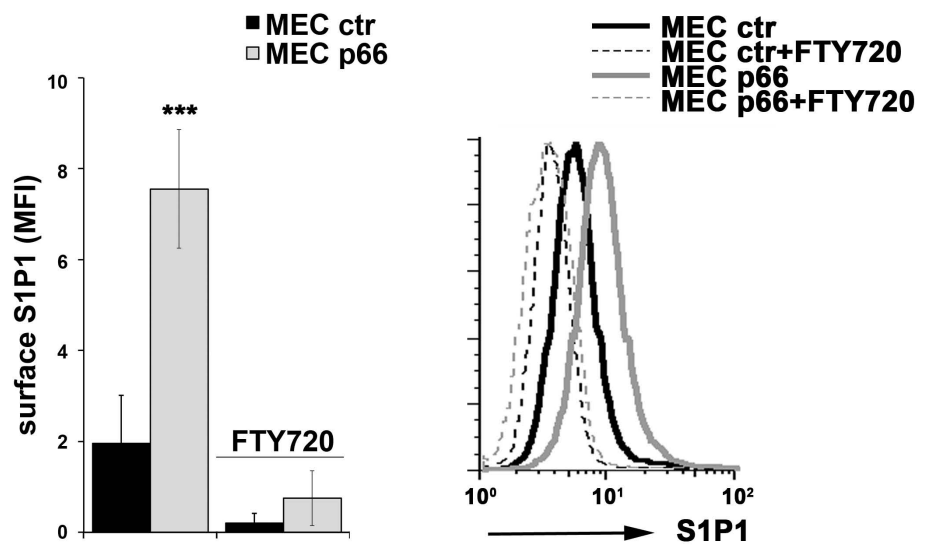

D
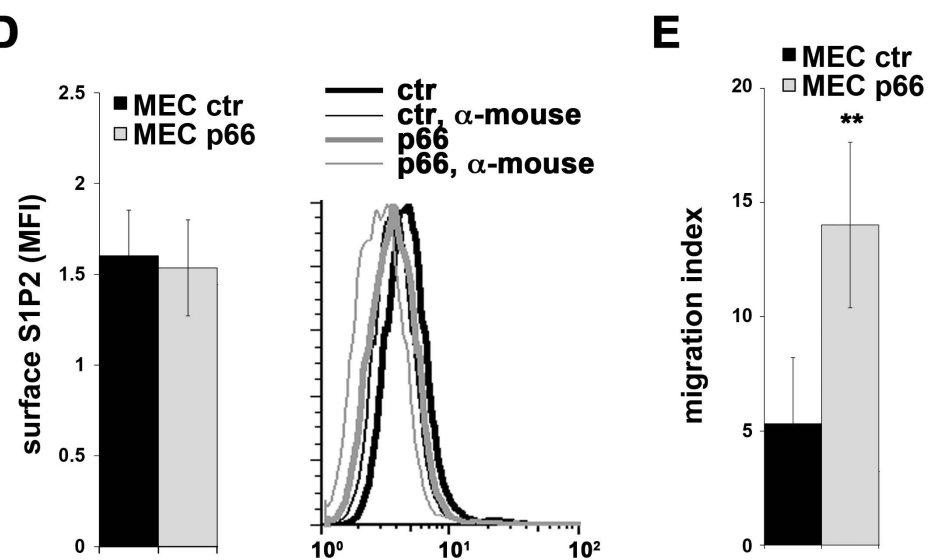
Figure 3

A

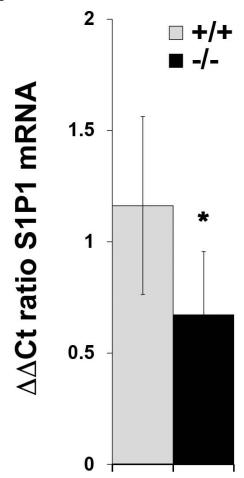

B

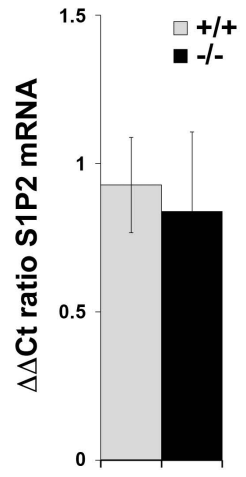

C

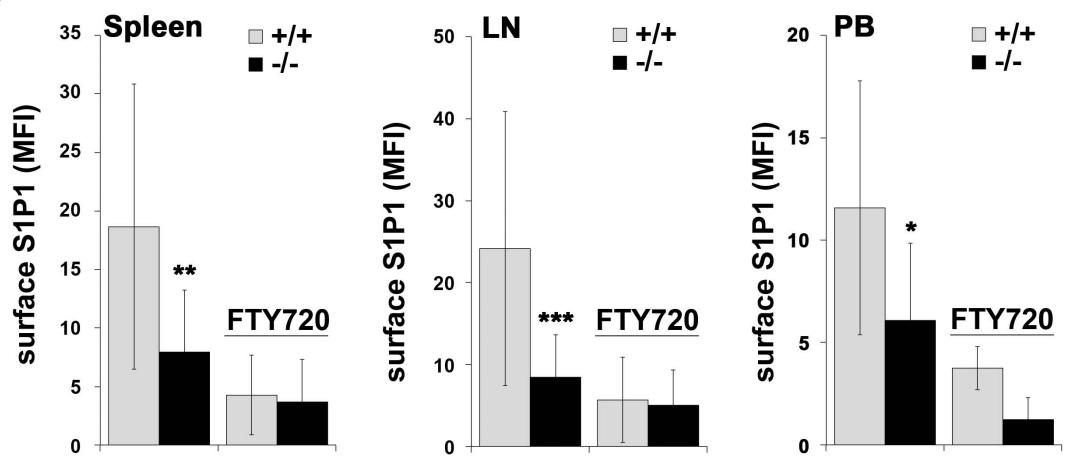

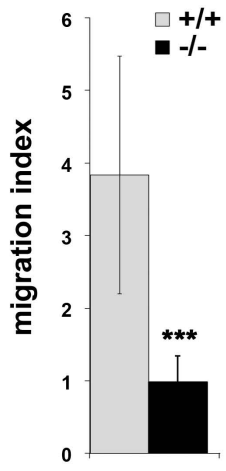


Figure 4

A

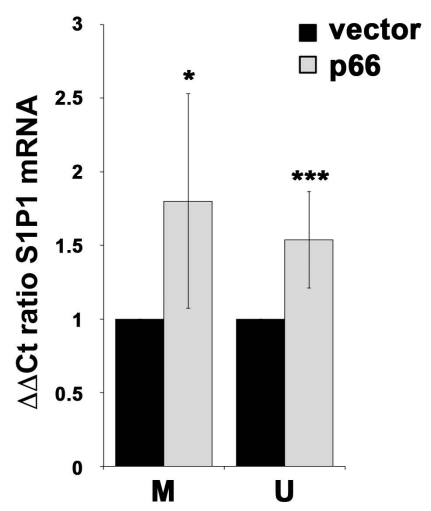

B

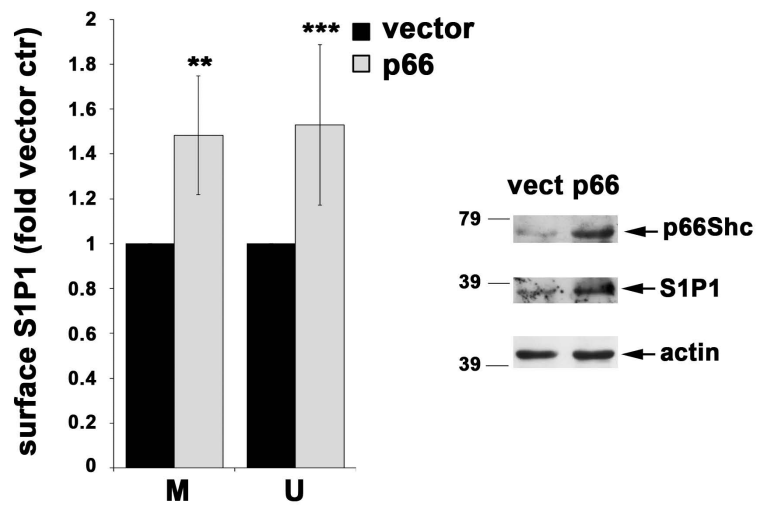


Figure 5

A

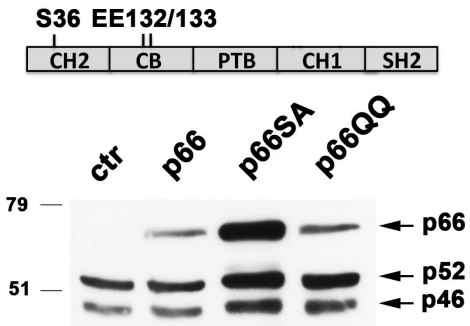

WB anti-Shc

39

WB anti-actin

C

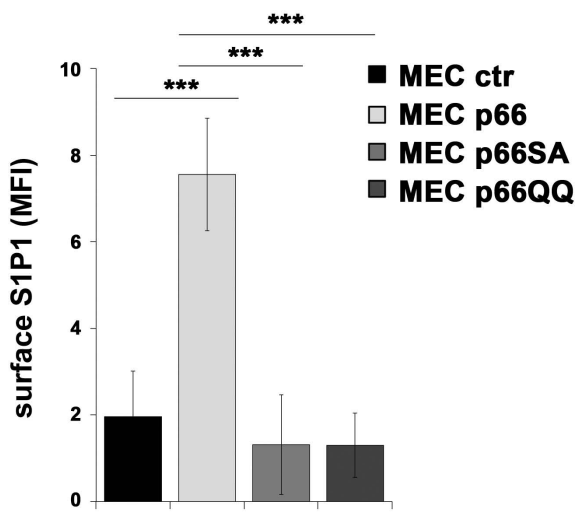

B

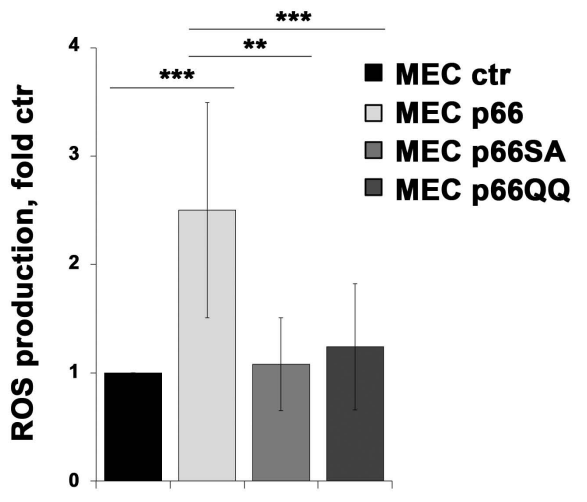

D

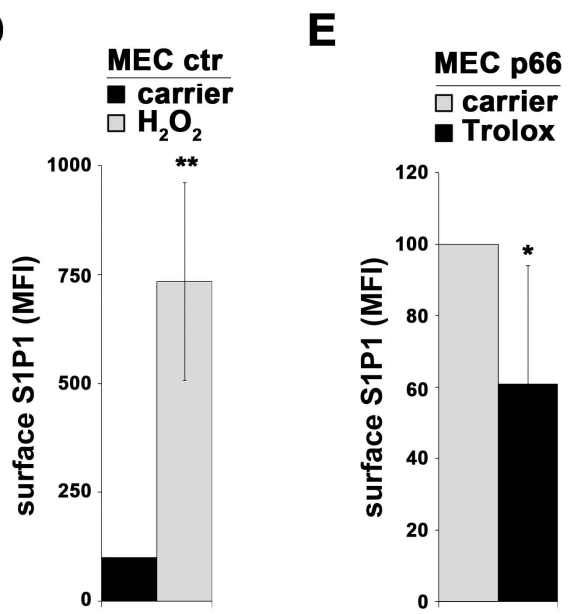


Figure 6

A

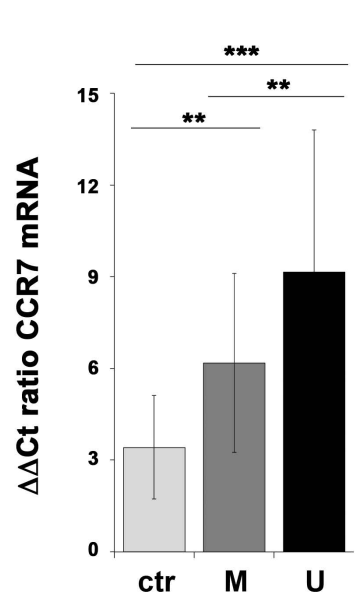

B

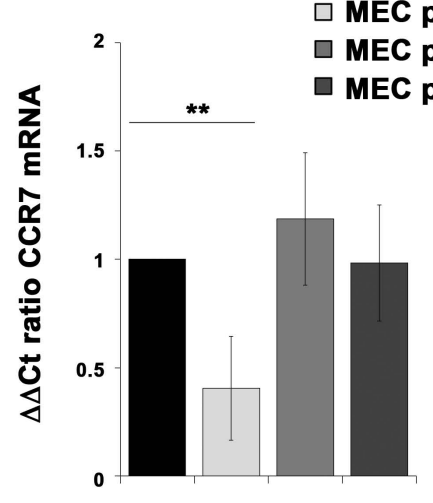

D
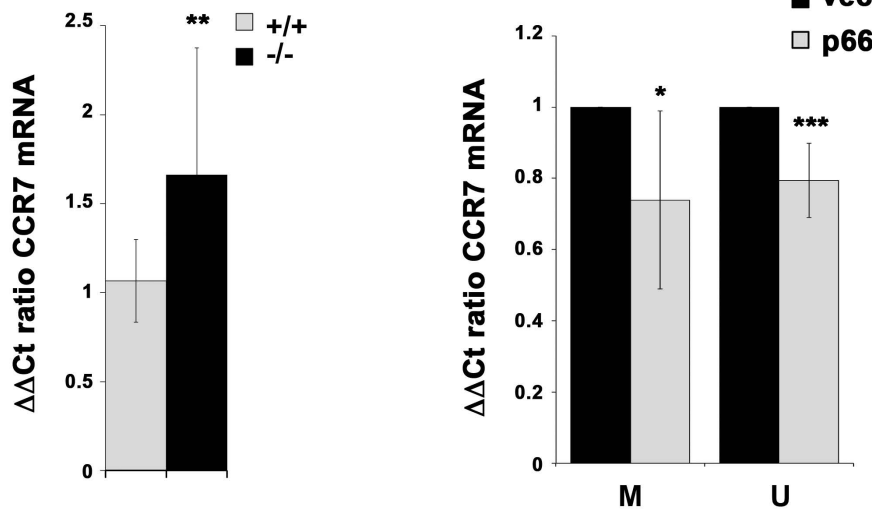